\title{
Impact of high-mobility-group A2 overexpression on epithelial-mesenchymal transition in pancreatic
} cancer

\author{
Jian Gong',* \\ Yuxiang Wang ${ }^{1, *}$ \\ Buping Jiang' \\ Bin Xu' \\ Chuanzhen $\mathrm{Hu}^{2,3}$ \\ 'Department of Hepato-Biliary- \\ Pancreatic Surgery, Shanghai Tenth \\ People's Hospital Affiliated to Tongji \\ University, Shanghai 200072, People's \\ Republic of China; ${ }^{2}$ Department of \\ Orthopaedic Surgery, Shanghai Tenth \\ People's Hospital Affiliated to Tongji \\ University, Shanghai 200072, People's \\ Republic of China; ${ }^{3}$ Institute of Bone \\ Tumor Affiliated to Tongji University \\ School of Medicine, Shanghai 200072 \\ People's Republic of China \\ *These authors contributed equally to \\ this work
}

Correspondence: Bin Xu

Department of Hepato-Biliary-Pancreatic Surgery, Shanghai Tenth People's Hospital Affiliated to Tongji University, 30I YanChang Middle Road, Shanghai 200072,

People's Republic of China

Tel +8613761622519

Email pfdbsxubin@163.com

Chuanzhen $\mathrm{Hu}$

Department of Orthopaedic Surgery, Shanghai Tenth People's Hospital Affiliated to Tongji University, 30I Yan-Chang Middle Road, Shanghai 200072, People's

Republic of China

Tel +8615821964045

Email seak2007@sjtu.edu.cn
This article was published in the following Dove Press journal: Cancer Management and Research

Background: Tumor metastasis causes high mortality in patients with malignancies. In carcinomas, overexpression of high-mobility-group A2 (HMGA2) in cancer cells would lead to tumor development and epithelial to mesenchymal transition (EMT), promoting metastasis. This study evaluated HMGA2 overexpression for its effects on pancreatic cancer (PC). Methods: HMGA2 protein levels were immunohistochemically assessed in human PC tissue specimens and evaluated for associations with patients' clinicopathological findings. In human PC CAPAN 1 cells after HMGA2 expression was silenced or overexpressed, Transwell migration and invasion assays were performed, and EMT marker levels (Ecadherin, N-cadherin and Vimentin) were determined by immunoblot.

Results: HMGA2 and Vimentin were found in $43 \%$ and $45 \%$ of PC tissue samples, respectively, while E-cadherin was absent in 60\%. HMGA2 expression, loss of E-cadherin and Vimentin expression were significantly associated with clinical stage, tumor differentiation and lymph node metastasis. More importantly, univariate and multivariate analysis demonstrated that HMGA2 expression is an independent prognostic factor for patients with pancreatic cancer. Meanwhile, HMGA2-silenced CAPAN 1 cells showed reduced migration and invasion ability while HMGA2-overexpressed CAPAN 1 cells showed increased migration and invasion ability. Increased E-cadherin (epithelial marker) and reduced N-cadherin and Vimentin (mesenchymal markers) were found in HMGA2-silenced cells, while reduced E-cadherin and increased N-cadherin and Vimentin were found in HMGA2-overexpressed cells. Furthermore, Snail and Zeb1 (transcriptional factors) were reduced in HMGA2-silenced cells and increased in HMGA2-overexpressed cells.

Conclusion: Our findings demonstrate that HMGA2 expression correlates with advanced tumor grades, lymph node metastasis and poor prognosis and may be a novel prognosis/ therapeutic marker for PC.

Keywords: HMGA2, human pancreatic cancer, prognosis, EMT

\section{Introduction}

The epithelial to mesenchymal transition (EMT) constitutes one of the most critical physiological processes in development biology, with the cells losing their epithelial features and gaining mesenchymal ones ${ }^{1}$. Recent reports have indicated EMT involvement in the malignant conversion of tumor cells; therefore, it reflects invasive or metastasizing properties in a variety of cancers 2 In EMT, cancer cells enhance their abilities of migration and invasion. The characteristic changes of EMT comprise the downregulation of the epithelial protein E-cadherin and 
increased levels of many mesenchymal molecules, including vimentin and N-cadherin. ${ }^{3,4}$ However, the exact mechanism of EMT remains to be clarified.

High-mobility group AT-hook (HMGA2) changes the DNA structure as an architectural factor, thereby regulating DNA-dependent activities, including transcription, replication and repair. ${ }^{5,6}$ HMGA2 is found at high levels in the embryo, but rarely expressed or absent in normal adult tissues, indicating its function in cell proliferation and differentiation7 However, several reports revealed that HMGA2 shows elevated amounts in cancer and is involved in tumor metastasis via EMT8 Overexpression of HMGA2 is significantly correlated with metastasis and poor prognosis in several cancers such as triple negative breast,9 ovarian,10 gastric, 11 colorectal $^{12}$ and non-small cell lung ${ }^{13}$ cancers. Strell et al. ${ }^{14}$ found HMGA2 is overexpressed in pancreatic cancer (PC), with levels showing tight associations with tumor differentiation, clinical stage and clinical prognosis. However, HMGA2 association with EMT features remains undefined. This work assessed HMGA2, E-cadherin and Vimentin levels in PC, and determined the association of HMGA2 with prognosis in PC patients. Finally, we further explored the mechanism by which HMGA2 affects EMT in vitro using PC cell lines.

\section{Materials and methods}

\section{Patients and specimens}

A total of 60 paraffin wax-embedded samples of pancreatic ductal adenocarcinoma and 9 samples from adjacent non-cancerous pancreatic tissues were obtained by Shanghai Tenth People's Hospital, from April 2002 to October 2006. This study had approval from the Ethics Committee of Shanghai Tenth People's Hospital (Shanghai, China). All tumors were classified and graded according to the WHO Classification of Tumors.

\section{Cells and culture}

Pancreatic cancer CAPAN 1 cells were provided by American Type Culture Collection (ATCC, USA), and maintained in Dulbecco's Modified Eagle Medium (DMEM) containing 10\% fetal bovine serum (FBS), penicillin $(100 \mathrm{U} / \mathrm{ml})$ and streptomycin $(100 \mathrm{mg} / \mathrm{ml})$ (all from Life Technologies, USA), at $37{ }^{\circ} \mathrm{C}$ in an incubator with $5 \% \mathrm{CO} 2$.

\section{Immunohistochemical staining}

Paraffin embedded pancreatic ductal adenocarcinoma specimens were sectioned and pretreated at $60^{\circ} \mathrm{C}(1 \mathrm{~h})$, followed by dewaxing in xylene, re-hydration with graded ethanol and incubation with citrate buffer (pH6.0) for antigen retrieval. Endogenous peroxidase activity was quenched for $30 \mathrm{~min}$ using $0.3 \% \quad \mathrm{H}_{2} \mathrm{O}_{2}$. Then, primary antibodies targeting HMGA2 diluted 1:100 in TBST (Cell Signaling Technology, USA) were added for overnight incubation at $4{ }^{\circ} \mathrm{C}$. The remaining procedures were carried out as directed by the manufacturer of the LSAB + kit (Dako, USA). Counterstaining was performed with hematoxylin. As proposed in a previous report(15), 0-5\%, 5-15\%, 15-50\% and $>50 \%$ cells stained indicated negative, weak, medium, and strong staining, respectively. Positively stained cancer cells were assessed in 5 high power fields for a total of 200 adjacent cells. Staining scoring was: $0,0 \% ; 1,1-25 \% ; 2,26-$ $49 \% ; 3,50-75 \%$; and 4, 76-100\%. Staining intensity was scored between 0 and 3 , corresponding to no, weak, moderate and strong staining, respectively. The overall score was obtained by multiplying the 2 individual scores (0-12). For statistical evaluation, specimens were assigned to negative (score $\leq 2$ ) or positive (score $>2$ ) groups. Slide assessment was performed by two blinded pathologists.

\section{Small interfering RNA-mediated knockdown}

Short interfering RNAs (siRNAs) targeting HMGA2 (HMGA2-siRNA1: CACAACAAGUCGUUCAGAA; and HMGA2-siRNA2: AGAGGCAGACCUAGGAAAU) and a GFP control siRNA (GFP-si) were provided by GenePharma (China).

Cells were transfected in six well plates with Lipofectamine 3000 (Life Technologies) as instructed by the manufacturer. Knockdown efficiency was assessed by immunoblot.

\section{Generation of HMGA2-overexpressing cells}

Plasmid pcDNA HMGA2 overexpressing HMGA2 or nontarget controls (pcDNA) were purchased from Shanghai Genechem Co., Ltd (China). We transfected the plasmid pcDNA-HMGA2 and non-target controls (pcDNA) into the CAPAN 1 cells with the Lipofectamine 3000 (Invitrogen). Forty-eight hours after transfection, the protein levels of HMGA2 were determined by western blotting.

\section{Quantitative RT-PCR}

Cell total RNA was obtained with TRIzol reagent (Life technologies) as instructed by the manufacturer. After 
reverse transcription, the resulting cDNA was amplified by quantitative RT-PCR (qRT-PCR) with a SYBR Premix Ex Tag kit (TaKaRa) on an ABI 7,500 Sequencing Detection System (Applied Biosystems, USA). Data were normalized to GAPDH expression. The following primers were used:

HMGA2, sense 5'-CAGGATGAGCGCACGCGGTGA GGGC-3' and antisense 5'-CCATTTCCTAGGTCTGCCTC TTGGC-3'; GAPDH, sense 5'-GGACCTGACCTGCCGTCT AG-3' and antisense 5'-GTAGCCCAGGATGCCCTTGA-3'.

\section{Immunoblot}

Cell lysis was performed with precooled RIPA buffer (Sigma, USA). After centrifugation $\left(12,000 \times \mathrm{g}, 4{ }^{\circ} \mathrm{C}\right.$, $20 \mathrm{~min}$ ) protein amounts were determined in the supernatant. Equal amounts of total protein were resolved by $10 \%$ SDS-PAGE and electro-transferred onto nitrocellulose membranes (Millipore). The membranes were blocked and incubated overnight at $4{ }^{\circ} \mathrm{C}$ with primary antibodies targeting HMGA2, E-cadherin, vimentin, Ncadherin, Snail, Zeb1 (Cell Signaling Technology, USA) and GAPDH (Santa Cruz, USA). All the primary antibodies were diluted to 1:1000 in TBST. After three washes with TBST, secondary antibodies were added for $1 \mathrm{~h}$ at room temperature. Visualization was performed on a LICOR infrared imaging system (LI-COR).

\section{Migration and invasion assays}

In migration assay, $5 \times 10^{4}$ pancreatic cancer cells in serum-free DMEM were plated in 24-well chambers (Corning Costar, USA) with no matrigel coating. In invasion assay, the upper chamber was pre-treated with matrigel (BD Bioscience) before addition of $5 \times 10^{4}$ cells in serum-free DMEM. After $24 \mathrm{~h}$ of culture, the cells crossing the membranes were incubated with $0.5 \%$ crystal violet and numbered using an inverted microscope. Cell numbers in 5 randomly selected high power fields were assessed.

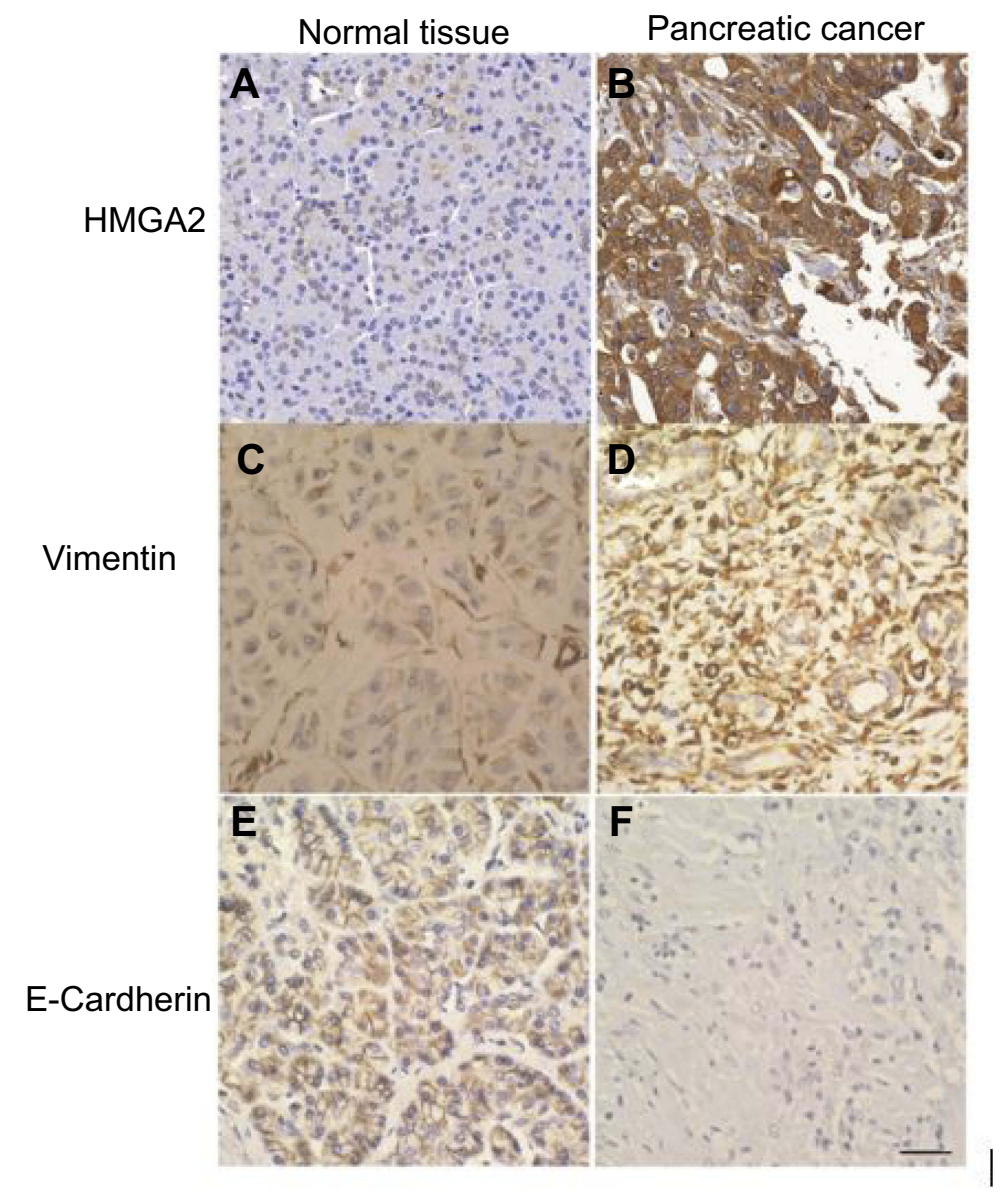

Figure I Immunohistochemical staining of HMGA2, E-cadherin and vimentin in normal pancreatic tissue and pancreatic cancer samples. (A) No expression of HMGA2 in the normal pancreatic tissue. (B) Positive signals of HMGA2 in pancreatic cancer. (C) No expression of vimentin in the normal pancreatic tissue. (D) Positive signals of vimentin in pancreatic cancer. (E) Positive signals of E-cadherin in the normal pancreatic tissue. (F): No expression of E-cadherin in pancreatic cancer. Scale bar=50 um. 


\section{Statistical analysis}

Data are mean \pm standard deviation (SD), and were assessed by two-tailed unpaired $t$-test and ANOVA for 2 or multiple groups. Associations of HMGA2, E-cadherin and vimentin protein levels with clinicopathological features were evaluated by the Pearson $\chi^{2}$ test. GraphPad Prism5 (GraphPad, USA) and SPSS 18.0 software (SPSS Inc., USA) was employed for all statistical analyses. The significance of various variables in the prognosis of the disease was assessed by the Cox proportional hazards regression model for univariate and multivariate analyses. $P<0.05$ indicated statistical significance.

\section{Results}

\section{HMGA2, E-cadherin and vimentin protein} levels in pancreatic cancer specimens and normal tissues

By immunohistochemical staining, we found that HMGA2 was mainly expressed in nuclei or the cytoplasm of human pancreatic cancer cells (Figure 1A and B), while vimentin was mainly found in the cytoplasm or membrane of tumor cells (Figure 1C and D). Meanwhile, E-cadherin showed positive signals in the membrane of normal cells from human normal pancreatic tissues (Figure 1E and F).

No HMGA2 expression was found in the 9 non-cancerous pancreatic specimens; however, positive signals for HMGA2 were observed in $43 \%(26 / 60)$ of pancreatic cancer tissue specimens $(P<0.001)$. A total of 36 PC cases $(60 \%)$ did not express E-cadherin among the 60 assessed, while the 9 normal pancreatic tissue samples had positive staining for E-cadherin $(P<0.001)$. Vimentin was expressed in 27 of the 60 cases $(45 \%)$ of pancreatic cancer tissue samples, whereas the 9 normal pancreatic tissue samples showed no expression.

\section{Associations of HMGA2, E-cadherin and vimentin protein amounts with clinicopathological properties of pancreatic cancer samples}

As shown in Table 1, HMGA2 levels in pancreatic cancer were tightly associated with clinical stage $(P=0.0285)$ and

Table I Association of HMGA2, E-cardherin and Vimentin expression with the clinicopathological features of 60 patients with pancreatic cancer

\begin{tabular}{|c|c|c|c|c|c|c|c|c|c|c|}
\hline \multirow[t]{2}{*}{ Characteristics } & \multirow[t]{2}{*}{ Cases } & \multicolumn{3}{|c|}{ HMGA2 expression } & \multicolumn{3}{|c|}{ E-cardherin expression } & \multicolumn{3}{|c|}{ Vimentin expression } \\
\hline & & Positive & Negative & $\boldsymbol{P}$ & Positive & Negative & $P$ & Positive & Negative & $\boldsymbol{P}$ \\
\hline Age & & & & 0.7563 & & & 0.6753 & & & 0.805 \\
\hline$\leq 60$ & 26 & 11 & 15 & & 12 & 14 & & 10 & 16 & \\
\hline $6 I-70$ & 16 & 6 & 10 & & 6 & 10 & & 9 & 7 & \\
\hline$>70$ & 18 & 9 & 9 & & 6 & 12 & & 8 & 10 & \\
\hline Gender & & & & 0.2981 & & & I & & & 0.4313 \\
\hline Male & 37 & 14 & 23 & & 15 & 22 & & 15 & 22 & \\
\hline Female & 23 & 12 & 11 & & 9 & 14 & & 12 & 11 & \\
\hline Treatment & & & & I & & & 0.2952 & & & 0.1932 \\
\hline No treatment & 33 & 15 & 18 & & 11 & 22 & & 12 & 21 & \\
\hline Gemcitabine & 27 & 12 & 15 & & 13 & 14 & & 15 & 12 & \\
\hline Clinical stage & & & & 0.0285 & & & 0.0344 & & & $0.005 I$ \\
\hline I & II & 1 & 10 & & 8 & 3 & & 2 & 9 & \\
\hline II & 43 & 21 & 22 & & 15 & 28 & & 19 & 24 & \\
\hline III/IV & 6 & 4 & 2 & & I & 5 & & 6 & 0 & \\
\hline Tumor & & & & 0.0419 & & & 0.0301 & & & 0.009 \\
\hline Differentiation & & & & & & & & & & \\
\hline Well differentiated & 8 & 1 & 7 & & 6 & 2 & & 2 & 6 & \\
\hline $\begin{array}{l}\text { Mediated } \\
\text { differentiated }\end{array}$ & 33 & 13 & 20 & & 14 & 19 & & 11 & 22 & \\
\hline Poor differentiated & 19 & 12 & 7 & & 4 & 15 & & 14 & 5 & \\
\hline Lymph node & & & & 0.0006 & & & 0.0326 & & & 0.0086 \\
\hline metastasis & & & & & & & & & & \\
\hline Yes & 26 & 18 & 8 & & 6 & 20 & & 17 & 9 & \\
\hline No & 34 & 8 & 26 & & 18 & 16 & & 10 & 24 & \\
\hline
\end{tabular}

Note: Statistically significant values are shown in bold. 

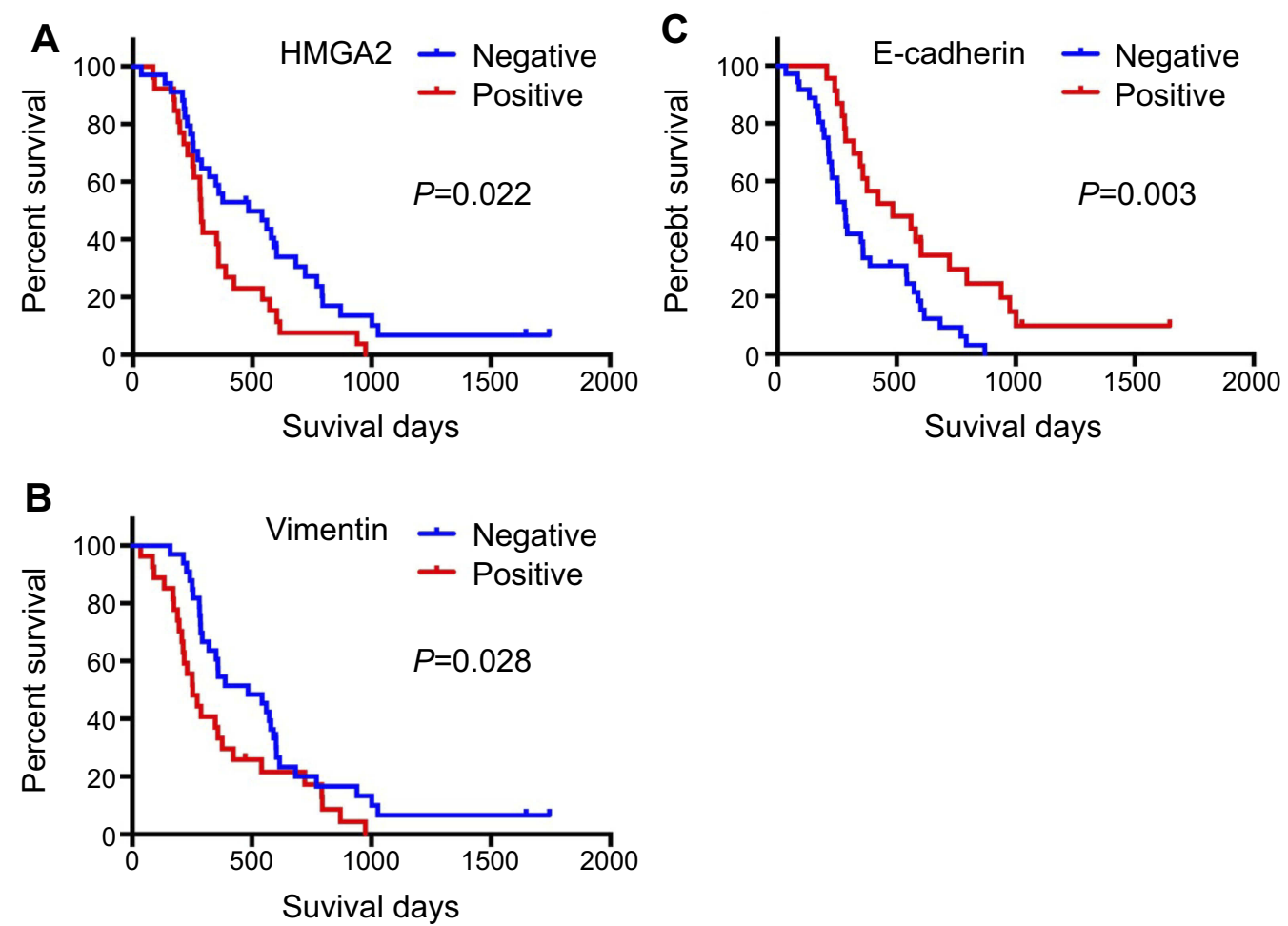

Figure 2 Survival curves of patients with HMGA2, E-cadherin and vimentin expression in pancreatic cancer. (A) Patients with high HMGA2 levels showed significantly worse survival than those with low expression $(P=0.022)$. (B) Patients with high vimentin levels showed significantly worse survival than those with low expression $(P=0.028)$. $(C)$ Patients with low E-cadherin levels had significantly worse survival than those with high expression $(P=0.003)$.

tumor differentiation $(P=0.0419)$. No E-cadherin expression was observed in $78.9 \%(15 / 19)$ of cases with poorly differentiated tumors and $83.3 \%(5 / 6)$ of cases with stage III/IV tumors, while positive signals were found in $75 \%$ $(6 / 8)$ of cases with well-differentiated tumors and $72.7 \%$ $(8 / 11)$ of cases with stage I tumors. Compared with stage I and well differentiated tumors, positive staining of vimentin was found in 51\% (25/49) of patients with stage II-IV tumors, and $48 \%(25 / 52)$ of patients with mediated and poorly differentiated tumors $(P=0.0051$ and $P=0.009$, respectively). In addition, HMGA2 and vimentin expression and E-cadherin absence were highly correlated with lymph node metastasis $(P=0.0006, \quad P=0.0086$ and $P=0.0326$ respectively). HMGA2, E-cadherin and vimentin expression had no associations with gender, age and gemcitabine treatment. (Table 1).

\section{Association between HMGA2 expression and patient survival}

The effect of HMGA2, E-cadherin and vimentin expression, on patient survival, were examined with Kaplan-
Meier analysis and the log-rank test. Patients with positive expression of HMGA2 and vimentin had markedly reduced overall survival (OS) compared with individuals not expressing the protein (Figure 2A and B). Meanwhile, patients with E-cadherin negative expression showed shorter OS compared with those positive expression (Figure 2C).

Then we used univariate and multivariate analyses to confirm whether HMGA2 expression was an independent predictor of a negative prognosis. As shown in Table 2, HMGA2 expression $(P=0.038)$ was an independent prognostic indicator for overall survival. (Table 2) Taken together, our data suggested that HMGA2 expression was obviously associated with the prognosis and also was an independent prognostic factor for patients with pancreatic cancer.

\section{HMGA2 promotes the migration and invasion in PC cells}

CAPAN 1 cells were transfected with siRNA against HMGA2 (siHMGA2) or scrambled siRNA (NC). The results showed that siHMGA2-1 and siHMGA2-2 starkly 
Table 2 Univariate and multivariate analysis of factors associated with overall- survival of patients with pancreatic cancer

\begin{tabular}{|c|c|c|c|c|c|c|c|}
\hline & \multirow[t]{2}{*}{ Cases } & \multicolumn{3}{|c|}{ Univariable } & \multicolumn{3}{|c|}{ Multivariable } \\
\hline & & HR & $95 \% \mathrm{Cl}$ & $\boldsymbol{P}$ & HR & $95 \% \mathrm{Cl}$ & $P$ \\
\hline \multicolumn{8}{|l|}{ Age } \\
\hline$\leq 60$ & 26 & 1 & & & & & \\
\hline$>60$ & 34 & 0.713 & $0.212-1.257$ & 0.383 & & & \\
\hline \multicolumn{8}{|l|}{ Gender } \\
\hline Male & 37 & I & & & & & \\
\hline Female & 23 & 0.461 & $0.292-1.321$ & 0.421 & & & \\
\hline \multicolumn{8}{|l|}{ Treatment } \\
\hline No treatment & 33 & 1 & & & 1 & & \\
\hline Gemcitabine & 27 & 0.342 & $0.127-0.764$ & 0.012 & 0.669 & $0.412-0.865$ & 0.032 \\
\hline \multicolumn{8}{|l|}{ Clinical stage } \\
\hline I & 11 & 1 & & & I & & \\
\hline II/III/IV & 49 & 4.239 & $2.126-8.432$ & 0.003 & 8.521 & $1.743-32.129$ & 0.026 \\
\hline \multicolumn{8}{|l|}{ Tumor Differentiation } \\
\hline Well/differentiated & 41 & I & & & I & & \\
\hline Poor differentiated & 19 & 4.569 & I.539-9.439 & 0.011 & 1.643 & $0.632-2.823$ & 0.727 \\
\hline \multicolumn{8}{|l|}{ Lymph node metastasis } \\
\hline No & 34 & 1 & & & 1 & & \\
\hline Yes & 26 & 3.153 & $1.356-6.539$ & 0.001 & 6.714 & $1.867-13.524$ & 0.015 \\
\hline \multicolumn{8}{|l|}{ HMGA2 expression } \\
\hline Negative & 34 & I & & & I & & \\
\hline Positive & 26 & 3.216 & $1.238-6.427$ & 0.001 & 5.712 & $1.128-13.572$ & 0.038 \\
\hline \multicolumn{8}{|l|}{ E-cadherin expression } \\
\hline Negative & 36 & I & & & 1 & & \\
\hline Positive & 24 & 0.394 & $0.164-0.715$ & 0.013 & 0.846 & $0.226-4.921$ & 0.561 \\
\hline \multicolumn{8}{|l|}{ Vimentin expression } \\
\hline Negative & 33 & I & & & I & & \\
\hline Positive & 27 & 4.936 & $1.133-8.316$ & 0.004 & 0.964 & $0.493-3.427$ & 0.096 \\
\hline
\end{tabular}

Note: Statistically significant values are shown in bold.

reduced HMGA2 protein amounts in PC cells in comparison with the $\mathrm{NC}$ group (Figure $3 \mathrm{~A}$ and $\mathrm{B}$ ). In order to increase the HMGA2 protein expression in CAPAN 1 cells, cells were transfected with Plasmid pcDNA HMGA2 or non-target controls (pcDNA). The results of Western blot in Figure 3C and D indicated that Plasmid pcDNA HMGA2 significantly increased the expression of HMGA2 protein in PC cells when compared to the NC group.

Importantly, HMGA2 knockdown cells showed decreased migration and invasion rates in comparison with the control group (Figure 4A and B), while HMGA2 overexpression cells showed increased migration and invasion rates in comparison with the control group (Figure 4C and D).

\section{HMGA2 promotes EMT}

Regulation migration and invasion abilities in HMGA2 silenced and overexpressed pancreatic cancer cells indicated HMGA2 contributes to EMT and metastasis. To further assess
HMGA2 significance in EMT, the levels of known EMTassociated proteins were assessed after HMGA2 silencing and overexpression by immunoblot. Interestingly, higher amounts of E-cadherin and lower $\mathrm{N}$-cadherin and vimentin levels were observed in HMGA2 knockdown PC cells while lower amounts of E-cadherin and higher $\mathrm{N}$-cadherin and vimentin levels were observed in HMGA2 overexpressed cells. In agreement, HMGA2 silencing resulted in downregulated Snail and Zeb1, two transcriptional markers involved in EMT, while HMGA2 overexpression resulted in increased Snail and Zeb 1. (Figure 5).

\section{Discussion}

In EMT, cancer cells lose their epithelial properties and gain mesenchymal ones, with increased malignant potential. $^{15,16}$ Signaling molecules, including Zeb1 and Snail, contribute to EMT, although the underpinning mechanisms remain largely unclear. ${ }^{17,18}$ HMGA2 
A

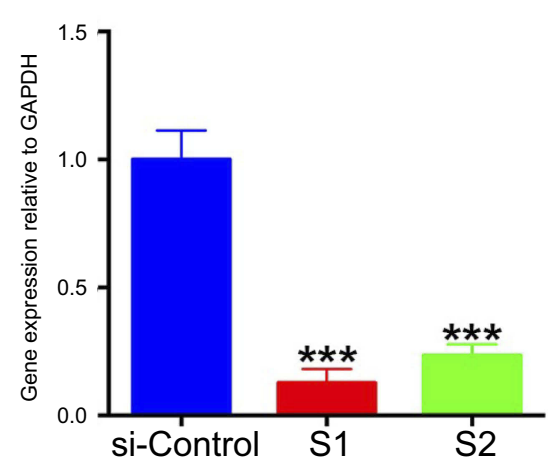

C

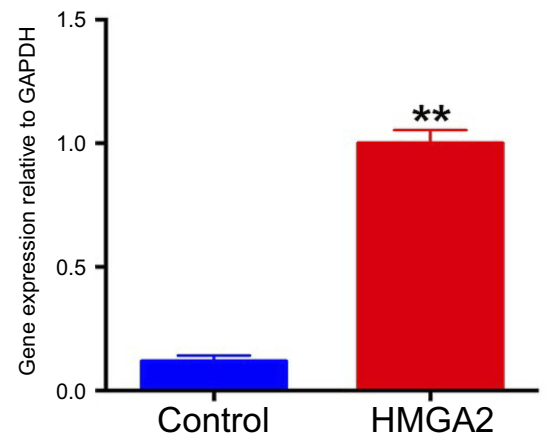

B

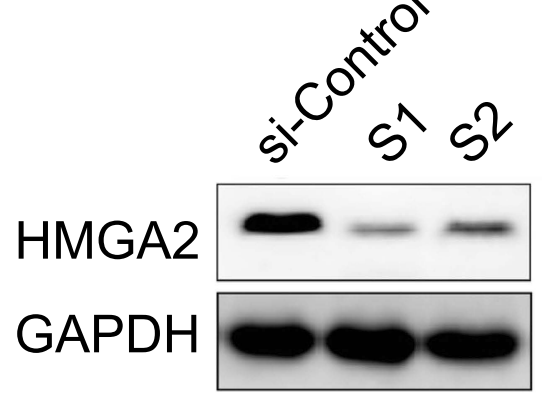

D

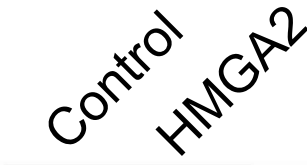

HMGA2

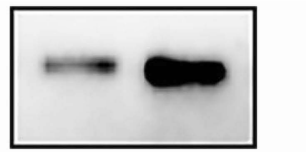

GAPDH

Figure 3 HMGA2 knockdown in pancreatic cancer CAPAN I cells using SiRNAs (HMGA2-SI and HMGA2-S2). (A) Quantitative real-time PCR and (B) Western blot data showing decreased HMGA2 mRNA and protein expression levels in both knockdown groups. ***P $<0.001$. HMGA2 over expressed in PC cells using plasmid pcDNA HMGA2 and non-target controls (PcDNA). (C) Quantitative real-time PCR and (D) Western blot data showing increased HMGA2 mRNA and protein expression levels in pcDNA HMGA2 group. **P<0.0I.

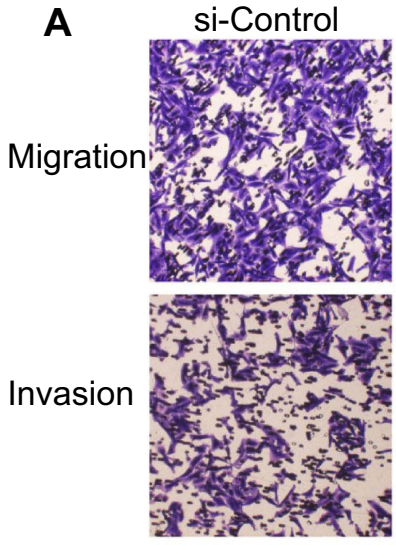

B

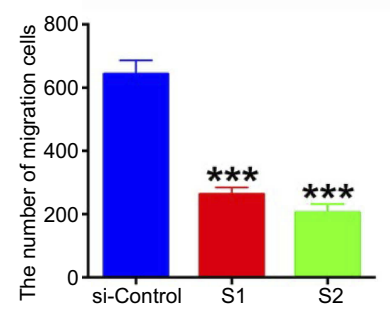

S1
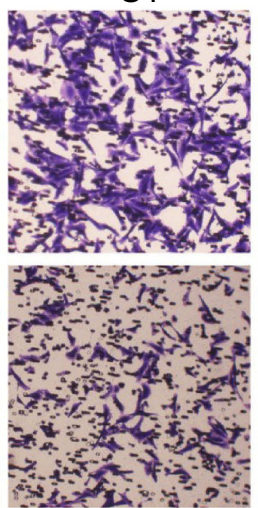

S2

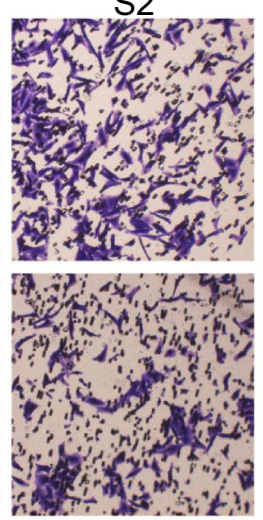

C

Migration

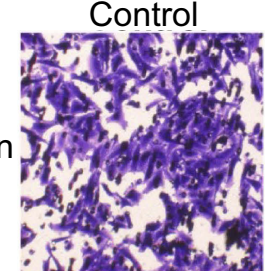

HMGA2

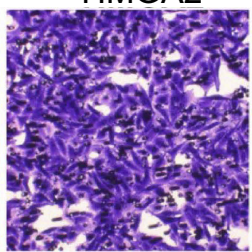

Invasion

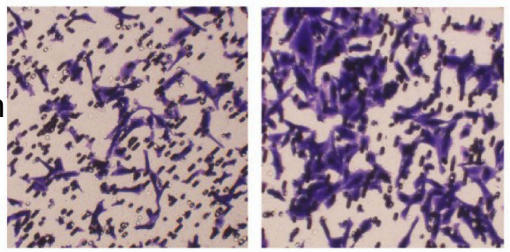

D
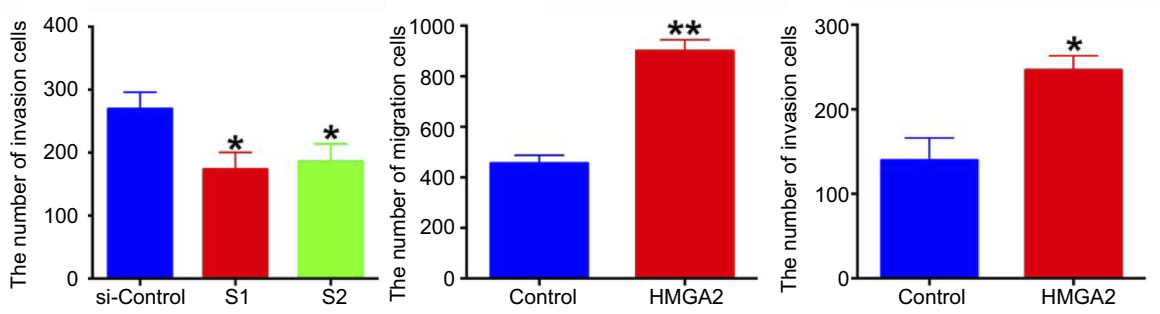

Figure 4 (A) Compared with control cells, migration and invasion abilities in HMGA2-SI and HMGA2-S2 cells were significantly inhibited (xI00). (B) Columns show the means of three independent experiments. ${ }^{*} P<0.05$, ${ }^{*} * *<0.00 \mathrm{I}$. (C) Compared with control cells, migration and invasion abilities in $\mathrm{HMGA} 2$ overexpressed cells were significantly enhanced $(x 100)$. (B) Columns show the means of three independent experiments. $* P<0.05, * * P<0.01$. 

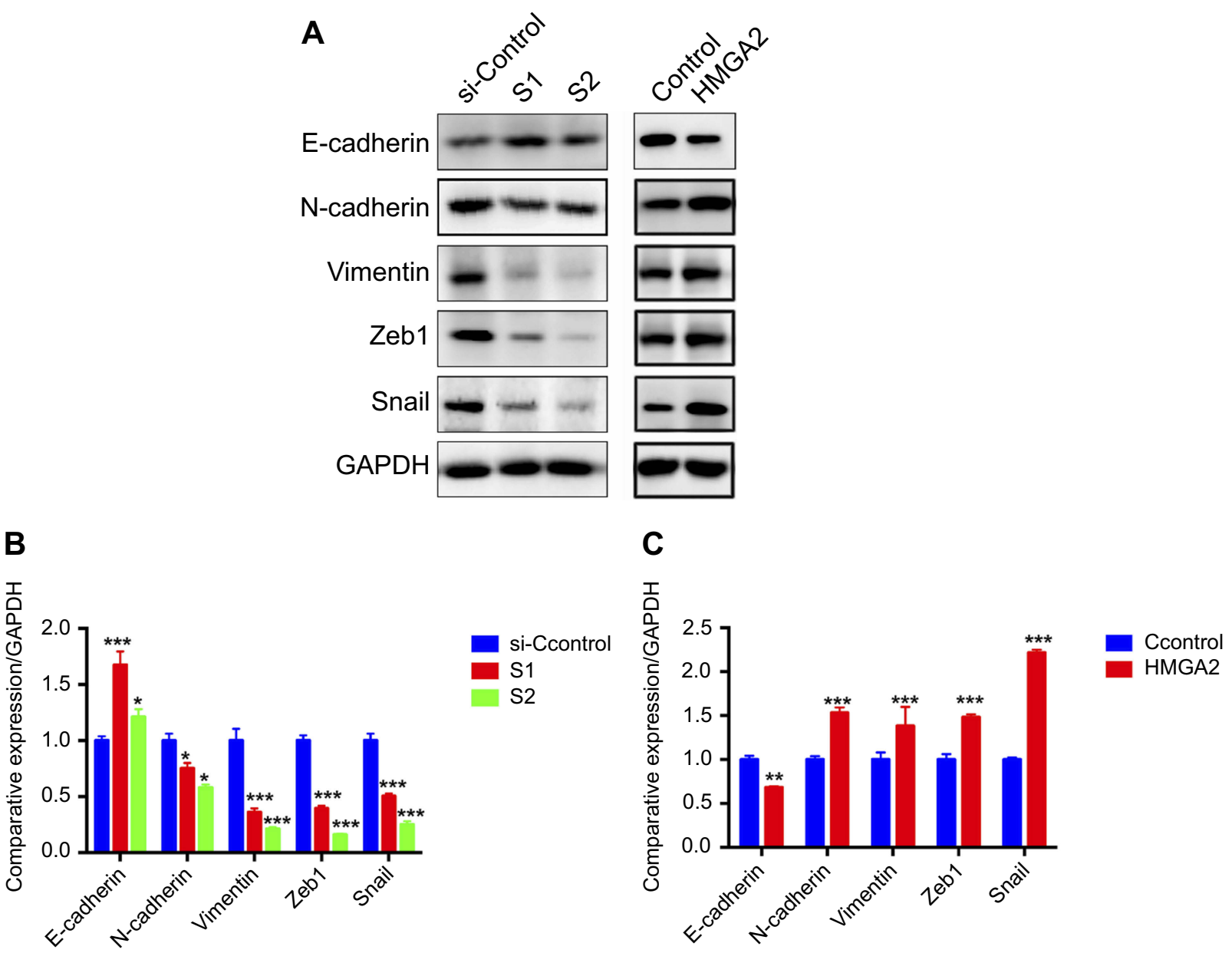

Figure 5 (A) Western blot for detecting EMT-associated protein markers in HMGA2 knockdown and overexpressed CAPAN I cells. (B) and (C) Semiquantitative histograms of Western blot results. $* P<0.05$, $* * P<0.01$, $* * * P<0.001$.

constitutes a novel factor affecting EMT in epithelial malignant tumors. ${ }^{19,20}$ HMGA2, found in several malignancies, is associated with tumor aggressiveness and poor prognosis. ${ }^{12,21}$ There are only two studies suggesting that HMGA2 is over-expressed in patients with pancreatic cancer, with its expression associated with patient's prognosis. ${ }^{14,22}$ However, the association of poor prognosis with HMGA2 over-expression remains undefined. Therefore, the current work assessed the clinical effects of HMGA2 as well as its association with EMT in PC.

EMT is critical for PC progression. ${ }^{23,24}$ In other carcinomas, HMGA2 contributes to cancer metastasis and progression via EMT induction; ${ }^{19,20}$ meanwhile, HMGA2 involvement in EMT in PC was reported by a single study 25 Therefore, HMGA2, E-cadherin and vimentin levels were assessed in 60 PC tissue specimens in this study, in an effort to evaluate HMGA2 effects on EMT features. As sown above, HMGA2 was negatively associated with E-cadherin amounts and displayed a positive correlation with vimentin levels. In addition, HMGA2 presence, loss of E-cadherin and vimentin expression were able to predict tumor progression and prognosis in pancreatic cancer. More importantly, by univariate and multivariate analyses we confirmed that HMGA2 expression was an independent prognostic indicator for overall survival of patients with pancreatic cancer. These findings indicated that HMGA2 suppression may prevent EMT.

To further define HMGA2 function during EMT, HMGA2 was silenced and overexpressed in human pancreatic cancer CAPAN 1 cells, and multiple EMT-associated proteins were detected. Zeb1, a transcription factor, promotes EMT by downregulating E-cadherin through interactions with its E-box18 Snail, another transcription factor, directly downregulates E-cadherin mRNA synthesis in EMT(17). We hypothesized that EMT-associated proteins are altered in expression after HMGA2 silencing. 
Indeed, HMGA2 silencing resulted in E-cadherin upregulation and N-cadherin, Zeb1, and Snail downregulation, while HMGA2 overexpression resulted in E-cadherin downregulation and N-cadherin, Zeb1, and Snail upregulation. In agreement, the migration and invasion abilities of CAPAN 1 cells were markedly reduced after HMGA2 silencing and significantly increased after HMGA2 overexpression. These findings indicated that HMGA2 contributes to EMT, and constitutes a molecular target for modulating malignancy in PC.

\section{Conclusion}

Overall, the current findings suggested that HMGA2 overexpression is critical in the late stage of PC and unveiled the association of HMGA2 with EMT in this malignancy. Additionally, HMGA2 expression markedly enhanced PC cell migration, invasion and EMT in vitro. Therefore, HMGA2 should be considered a novel biomarker for prognosis and may be a potential therapeutic target in pancreatic cancer.

\section{Availability of data and materials}

All datasets supporting the conclusions of this work are included in the article.

\section{Informed consent}

All patients provided written informed consent in compliance with the Declaration of Helsinki.

\section{Acknowledgments}

This project was funded in part by Shanghai Tenth People's Hospital (SYGZRPY2017021 and SYGZRPY2017OO4) and National Nature Science Foundation of China (NSFC) (NO. 81802840).

\section{Disclosure}

The authors report no conflicts of interest in this work.

\section{References}

1. Kim DH, Xing T, Yang Z, Dudek R, Lu Q, Chen YH. Epithelial mesenchymal transition in embryonic development, tissue repair and cancer: a comprehensive overview. J Clin Med. 2017;7:1.

2. Creighton CJ, Gibbons DL, Kurie JM. The role of epithelial-mesenchymal transition programming in invasion and metastasis: a clinical perspective. Cancer Manag Res. 2013;5:187-195. doi:10.2147/CMAR.S35171

3. Bendinelli P, Maroni P, Matteucci E, Desiderio MA. Hgf and tgfbeta1 differently influenced wwox regulatory function on twist program for mesenchymal-epithelial transition in bone metastatic versus parental breast carcinoma cells. Mol Cancer. 2015;14:112. doi:10.1186/s12943014-0278-9
4. Wang KH, Kao AP, Chang CC, Lin TC, Kuo TC. Bisphenol a-induced epithelial to mesenchymal transition is mediated by cyclooxygenase- 2 up-regulation in human endometrial carcinoma cells. Reprod Toxicol. 2015;58:229-233. doi:10.1016/j.reprotox.2015.10.011

5. Fujikane R, Komori K, Sekiguchi M, Hidaka M. Function of highmobility group a proteins in the dna damage signaling for the induction of apoptosis. Sci Rep. 2016;6:31714. doi:10.1038/srep31714

6. Chen B, Young J, Leng F. Dna bending by the mammalian highmobility group protein at hook 2. Biochemistry. 2010;49:1590-1595. doi:10.1021/bi901881c

7. Li O, Li J, Droge P. Dna architectural factor and proto-oncogene hmga2 regulates key developmental genes in pluripotent human embryonic stem cells. FEBS Lett. 2007;581:3533-3537. doi:10.1016/j.febslet.2007.06.072

8. Morishita A, Zaidi MR, Mitoro A, et al. Hmga2 is a driver of tumor metastasis. Cancer Res. 2013;73:4289-4299. doi:10.1158/0008-5472. CAN-12-3848

9. Wu J, Zhang S, Shan J, et al. Elevated hmga2 expression is associated with cancer aggressiveness and predicts poor outcome in breast cancer. Cancer Lett. 2016;376:284-292. doi:10.1016/j. canlet.2016.04.005

10. Wu J, Lai M, Shao C, Wang J, Wei JJ. Stc2 overexpression mediated by hmga2 is a biomarker for aggressiveness of high-grade serous ovarian cancer. Oncol Rep. 2015;34:1494-1502. doi:10.3892/ or. 2015.4120

11. Motoyama K, Inoue H, Nakamura Y, Uetake H, Sugihara K, Mori M. Clinical significance of high mobility group a2 in human gastric cancer and its relationship to let-7 microrna family. Clin Cancer Res. 2008;14:2334-2340. doi:10.1158/1078-0432.CCR07-4667

12. Wang X, Liu X, Li AY, et al. Overexpression of hmga2 promotes metastasis and impacts survival of colorectal cancers. Clin Cancer Res. 2011;17:2570-2580. doi:10.1158/1078-0432.CCR-10-2542

13. Guo X, Shi J, Wen Y, et al. Increased high-mobility group a2 correlates with lymph node metastasis and prognosis of non-small cell lung cancer. Cancer Biomark. 2018;21:547-555. doi:10.3233/ CBM-170401

14. Strell C, Norberg KJ, Mezheyeuski A, et al. Stroma-regulated hmga2 is an independent prognostic marker in pdac and aac. $\mathrm{Br} J$ Cancer. 2017;117:65-77. doi:10.1038/bjc.2017.140

15. Chen D, Hu C, Wen G, Yang Q, Zhang C, Yang H. Downregulated sox4 expression suppresses cell proliferation, migration, and induces apoptosis in osteosarcoma in vitro and in vivo. Calcif Tissue Int. 2018;102:117-127. doi:10.1007/s00223-017-0340-x

16. Nishiyama M, Tsunedomi R, Yoshimura K, et al. Metastatic ability and the epithelial-mesenchymal transition in induced cancer stemlike hepatoma cells. Cancer Sci. 2018. doi:10.1111/cas.13527

17. Campbell K, Lebreton G, Franch-Marro X, Casanova J. Differential roles of the drosophila emt-inducing transcription factors snail and serpent in driving primary tumour growth. PLoS Genet. 2018;14: e1007167. doi:10.1371/journal.pgen.1007664

18. Krebs AM, Mitschke J, Lasierra LM, et al. The emt-activator zeb1 is a key factor for cell plasticity and promotes metastasis in pancreatic cancer. Nat Cell Biol. 2017;19:518-529. doi:10.1038/ncb3513

19. Kou B, Liu W, Tang X, Kou Q. Hmga2 facilitates epithelialmesenchymal transition in renal cell carcinoma by regulating the tgf-beta/smad2 signaling pathway. Oncol Rep. 2018;39:101-108. doi:10.3892/or.2017.6091

20. Li W, Wang Z, Zha L, Kong D, Liao G, Li H. Hmga2 regulates epithelial-mesenchymal transition and the acquisition of tumor stem cell properties through twist1 in gastric cancer. Oncol Rep. 2017;37:185-192. doi:10.3892/or.2016.5255

21. Xia YY, Yin L, Tian H, et al. Hmga2 is associated with epithelialmesenchymal transition and can predict poor prognosis in nasopharyngeal carcinoma. OncoTargets Ther. 2015;8:169-176. doi:10.2147/OTT. S74397 
22. Piscuoglio S, Zlobec I, Pallante P, et al. Hmga1 and hmga2 protein expression correlates with advanced tumour grade and lymph node metastasis in pancreatic adenocarcinoma. Histopathology. 2012;60:397-404. doi:10.1111/j.1365-2559.2011. 04121.x

23. Cano CE, Motoo Y, Iovanna JL. Epithelial-to-mesenchymal transition in pancreatic adenocarcinoma. ScientificWorldJournal. 2010;10:19471957. doi:10.1100/tsw.2010.183
24. Liu H, Wang H, Liu X, Yu T. Mir-1271 inhibits migration, invasion and epithelial-mesenchymal transition by targeting zeb1 and twist1 in pancreatic cancer cells. Biochem Biophys Res Commun. 2016;472:346-352. doi:10.1016/j.bbrc.2016.02.096

25. Watanabe S, Ueda Y, Akaboshi S, Hino Y, Sekita Y, Nakao M. Hmga2 maintains oncogenic ras-induced epithelial-mesenchymal transition in human pancreatic cancer cells. Am J Pathol. 2009;174:854-868. doi:10.2353/ajpath.2009.080523

\section{Publish your work in this journal}

Cancer Management and Research is an international, peer-reviewed open access journal focusing on cancer research and the optimal use of preventative and integrated treatment interventions to achieve improved outcomes, enhanced survival and quality of life for the cancer patient.
The manuscript management system is completely online and includes a very quick and fair peer-review system, which is all easy to use. Visit http://www.dovepress.com/testimonials.php to read real quotes from published authors. 